Technical note

\title{
A new cone-beam computed tomography dosimetry method providing optimal measurement positions: A Monte Carlo study
}

\author{
Tomonobu Haba $^{\text {a, }}$, Keisuke Yasui ${ }^{a}$, Yasunori Saito ${ }^{\mathrm{b}}$, Masanao Kobayashi ${ }^{\mathrm{a}}$, Shuji Koyama ${ }^{\mathrm{c}}$ \\ ${ }^{\text {a }}$ Faculty of Radiological Technology, School of Medical Science, Fujita Health University, 1-98 Dengakugakubo, Kutsukake-cho, Toyoake, Aichi 470-1192, Japan \\ ${ }^{\mathrm{b}}$ Department of Radiology, Fujita Health University Hospital, 1-98 Dengakugakubo, Kutsukake-cho, Toyoake, Aichi 470-1192, Japan \\ ${ }^{\mathrm{c}}$ Brain \& Mind Research Center, Nagoya University, 1-1-20 Daiko-Minami, Higashi-ku, Nagoya, Aichi, Japan
}

\section{A R T I C L E I N F O}

\section{Keywords:}

Cone-beam CT dosimetry

Weighted CTDI

Monte Carlo simulation

\begin{abstract}
A B S T R A C T
Purpose: The conventional weighted computed tomography dose index $\left(\mathrm{CTDI}_{\mathrm{w}}\right)$ may not be suitable for conebeam computed tomography (CBCT) dosimetry because a cross-sectional dose distribution is angularly inhomogeneous owing to partial angle irradiations. This study was conducted to develop a new dose metric $\left(\mathrm{f}(0)^{\mathrm{CB}}{ }_{\mathrm{w}}\right)$ for CBCT dosimetry to determine a more accurate average dose in the central cross-sectional plane of a cylindrical phantom using Monte Carlo simulations.

Methods: First, cross-sectional dose distributions of cylindrical polymethyl methacrylate phantoms over a wide range of phantom diameters $(8-40 \mathrm{~cm})$ were calculated for various CBCT scan protocols. Then, by obtaining linear least-squares fits of the full datasets of the cross-sectional dose distributions, the optimal radial positions, which represented measurement positions for the average phantom dose, were determined. Finally, the $f(0)^{\mathrm{CB}}{ }_{w}$ method was developed by averaging point doses at the optimal radial positions of the phantoms. To demonstrate its validity, the relative differences between the average doses and each dose index value were estimated for the devised $\mathrm{f}(0)^{\mathrm{CB}}{ }_{\mathrm{w}}$, conventional $\mathrm{CTDI}_{\mathrm{w}}$, and Haba's $\mathrm{CTDI}_{\mathrm{w}}$ methods, respectively.

Results: The relative differences between the average doses and each dose index value were within 4.1\%, 16.7\%, and $11.9 \%$ for the devised, conventional $\mathrm{CTDI}_{\mathrm{w}}$, and Haba's $\mathrm{CTDI}_{\mathrm{W}}$ methods, respectively.

Conclusions: The devised $f(0)^{C B}{ }_{w}$ value was calculated by averaging four "point doses" at $90^{\circ}$ intervals and the optimal radial positions of the cylindrical phantom. The devised method can estimate the average dose more accurately than the previously developed $\mathrm{CTDI}_{\mathrm{W}}$ methods for CBCT dosimetry.
\end{abstract}

\section{Introduction}

Cone-beam computed tomography (CBCT) is an essential tool that is used in medical applications such as interventional radiology and image-guided radiation therapy. Similar to X-ray CT scanning, an X-ray tube rotates around a patient to produce a wide longitudinal beam with partial angle irradiation (e.g., $210^{\circ}$ ) during CBCT scanning. For many years, dose evaluation of CT examination has been performed using the volume CT dose index $\left(\mathrm{CTDI}_{\mathrm{vol}}\right)$, which is based on the weighted CTDI $\left(\mathrm{CTDI}_{\mathrm{w}}\right)$ and $\mathrm{CTDI}_{100}$ parameters [1]. $\mathrm{CTDI}_{100}$ measurements are conducted utilizing a 10-cm-long CT ionization chamber and a cylindrical polymethyl methacrylate (PMMA) phantom (CTDI phantom) with a length of $15 \mathrm{~cm}$. Because the concept of $\mathrm{CTDI}_{100}$ was developed for the conventional narrow longitudinal beam CT, $\mathrm{CTDI}_{100}$ is no longer applicable for CBCT dose estimation, owing to its large longitudinal beam width [2].

To overcome the disadvantages of the $\mathrm{CTDI}_{100}$ method, the International Electrotechnical Commission (IEC) [3] and American Association of Physicists in Medicine (AAPM) (task group 111) proposed new CBCT dosimetry metrics [4]. In particular, the former proposed to measure $C T D I_{I E C}$ by multiplying $C T D I_{100 \text {, ref }}$ corresponding to the $C T D I_{100}$ value obtained for a reference narrow longitudinal beam $(\leq 40 \mathrm{~mm})$ by a correction factor. The latter represents a ratio of the two CTDI $I_{\text {free-in-air }}$ values determined for the studied wide longitudinal beam and reference narrow longitudinal beam [3]. Meanwhile, the AAPM task group 111 proposed to measure a "point dose $f(0)$ " using a small ionization chamber with a length of $23 \mathrm{~mm}$ and long cylindrical phantom with a length of $45 \mathrm{~cm}$ to implement the full radiation scattering conditions [4].

Abuhaimed et al. investigated the efficiencies of the CTDI ${ }_{\text {IEC }}$ and point dose approaches as CBCT dose metrics $[5,6]$. They concluded that

\footnotetext{
* Corresponding author.

E-mail address: habatomo@fujita-hu.ac.jp (T. Haba).
} 
Table 1

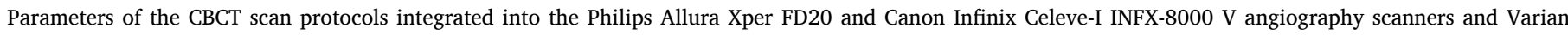
TrueBeam linear accelerator.

\begin{tabular}{|c|c|c|c|c|c|c|c|c|}
\hline \multirow{2}{*}{$\begin{array}{l}\text { Scan } \\
\text { region }\end{array}$} & \multirow{2}{*}{$\begin{array}{l}\text { Scanner } \\
\text { model }\end{array}$} & \multirow{2}{*}{$\begin{array}{l}\text { Source-isocenter- } \\
\text { distance }[\mathrm{cm}]\end{array}$} & \multirow{2}{*}{$\begin{array}{l}\text { Termed scan } \\
\text { protocol }\end{array}$} & \multirow{2}{*}{$\begin{array}{l}\text { Rotation angle (Covered } \\
\text { area) [deg.] }\end{array}$} & \multirow{2}{*}{$\begin{array}{l}\text { Tube voltage } \\
{[\mathrm{kV}]}\end{array}$} & \multirow{2}{*}{$\begin{array}{l}\text { Exposure } \\
{[\mathrm{mAs}]}\end{array}$} & \multicolumn{2}{|c|}{ Beam width $[\mathrm{mm}]$} \\
\hline & & & & & & & $\begin{array}{l}\text { Longitudinal } \\
\text { direction }\end{array}$ & $\begin{array}{l}\text { Lateral } \\
\text { direction }\end{array}$ \\
\hline \multirow[t]{6}{*}{ Head } & Allura Xper & 81 & Head-prop & $75-285(210)$ & 81 & 146 & 198 & 256 \\
\hline & FD20 & & Head-roll & $90-270(180)$ & 82 & 142 & & \\
\hline & & & Head-XperCT & $77-284(207)$ & 120 & 250 & & \\
\hline & Infinix & 70 & Head-3D & $79-281(202)$ & 90 & 265 & 190 & 190 \\
\hline & Celeve-I & & Head-AlphaCT & $72-288(216)$ & 81 & 2969 & & \\
\hline & TrueBeam & 100 & Head & $90-290(200)$ & 100 & 150 & 185 & 262 \\
\hline \multirow[t]{4}{*}{ Body } & Allura Xper & 81 & Body-prop & $75-285(210)$ & 104 & 496 & 198 & 256 \\
\hline & FD20 & & Body-roll & $90-270(180)$ & 107 & 498 & & \\
\hline & & & Body-XperCT & $76-284(208)$ & 123 & 325 & & \\
\hline & TrueBeam & 100 & $\begin{array}{l}\text { Body-spotlight (Fan } \\
\text { type: full) }\end{array}$ & $90-290(200)$ & 125 & 750 & 185 & 262 \\
\hline
\end{tabular}

the point dose approach is suitable for CBCT dosimetry because the obtained point dose values were closer to the $C T D I_{\infty}$ values than to the $C T D I_{I E C}$ values [6]. In addition, the $\mathrm{CTDI}_{100}$ method uses a 10 -cm-long CT ionization chamber to estimate a point dose at the center of a scan length for CT scanning with table translation [7]. Hence, this method cannot be employed for CBCT scanning without table translation because such an ionization chamber would measure an average dose over a length of $10 \mathrm{~cm}$ [2]. Because CTDI $_{\text {IEC }}$ is a modified CTDI $_{100}$ metric, the point dose approach recommended by AAPM task group 111 is consistent with the CTDI concept and represents a suitable dose metric for CBCT scanning without table translation $[6,8,9]$.

Although the above-mentioned studies mainly focused on dose assessment along the longitudinal beam direction, it is also important to investigate the cross-sectional dose profile of the CTDI phantom. The concept of the $\mathrm{CTDI}_{\mathrm{W}}$ metric is to provide the average dose in the central cross-sectional plane of the phantom [10]. To ensure accurate dose assessment during CT scanning, several $\mathrm{CTDI}_{\mathrm{W}}$ methods described by the following equations have been developed:

$$
\begin{aligned}
& C T D I_{w}^{C}=\frac{1}{3} \cdot C T D I_{\text {center }}+\frac{2}{3} \cdot C T D I_{\text {periphery }} \\
& C T D I_{w}^{B}=\frac{1}{2} \cdot C T D I_{\text {center }}+\frac{1}{2} \cdot C T D I_{\text {periphery }} \\
& C T D I_{w}^{H}=\frac{3}{8} \cdot C T D I_{\text {center }}+\frac{5}{8} \cdot C T D I_{\text {periphery }}
\end{aligned}
$$

where $C T D I_{\text {center }}$ and $C T D I_{\text {periphery }}$ are measured at the center and periphery ( $1 \mathrm{~cm}$ below the surface) of the CTDI phantom, respectively. $\mathrm{CTDI}_{\mathrm{w}}^{\mathrm{C}}$ has been adopted as the current international standard [10]. The $\mathrm{CTDI}^{\mathrm{B}}{ }_{\mathrm{w}}$ term proposed by Bakalyar, which is based on a second-order Taylor series expansion, is used to estimate the average dose of the CTDI phantom [11]. Haba et al. developed a new $\mathrm{CTDI}^{\mathrm{H}}{ }_{\mathrm{w}}$ metric using Monte Carlo simulations and the linear least-squares technique [12]. The differences between the average dose and $C T D I_{w}$ values were within $16 \%\left(C T D I^{C}{ }_{w}\right),-12 \%\left(C T D I^{B}{ }_{w}\right)$, and $-6 \%\left(C T D I^{H}{ }_{w}\right)$ for various phantom diameters $(8-40 \mathrm{~cm})$ and tube voltages $(80,100,120$, and $135 \mathrm{kV})$ in a Canon Aquilion ONE CT scanner; hence, the $\mathrm{CTDI}^{\mathrm{H}}{ }_{\mathrm{w}}$ equation can determine a more accurate average dose than those computed by the $\mathrm{CTDI}^{\mathrm{C}}{ }_{\mathrm{w}}$ and $\mathrm{CTDI}^{\mathrm{B}}{ }_{\mathrm{w}}$ equations [12].

The $\mathrm{CTDI}_{\mathrm{w}}$ equation is also used for CBCT dose evaluation, as proposed in the IEC 60601-2-44 and AAPM task group 111 reports [5,6]. In addition to being a standardized way of reporting dose indexes, $\mathrm{CTDI}_{\mathrm{w}}$ serves as an important intermediate quantity for calculating the patient size-specific dose estimate (SSDE) [13]. As such, it is important to estimate an accurate average dose in the central cross-sectional plane of the CTDI phantom for CBCT scans. However, the suitability of the $\mathrm{CTDI}^{\mathrm{H}}{ }_{\mathrm{w}}$ equation has not been validated for CBCT scans, which involve partial angle irradiations. In addition, the conventional five measurement positions (the center and four peripheries of the $90^{\circ}$ interval) of the CTDI phantom may be inappropriate for CBCT dosimetry because a crosssectional dose distribution is angularly inhomogeneous owing to partial angle irradiations (not a full $360^{\circ}$ scan). The conventional peripheral positions of the $90^{\circ}$ interval are based on the premise that the $x$-ray tube rotates with a full $360^{\circ}$ scan [10], and measurement positions at fine intervals (e.g., $45^{\circ}$ or $30^{\circ}$ ) may be appropriate to estimate an average dose of the phantom for CBCT scans.

Therefore, this study was conducted to devise a new dose metric ( $\mathrm{f}$ $(0)^{\mathrm{CB}}{ }_{\mathrm{w}}$ ) for CBCT dosimetry to determine a more accurate average dose of the CTDI phantom. It incorporates new phantom measurement positions instead of the five conventional ones, as well as the "point dose" method proposed in the AAPM task group 111 report. For this purpose, we first calculated cross-sectional dose distributions of the CTDI phantom for various CBCT protocols integrated into two angiography scanners and one linear accelerator by performing Monte Carlo simulations. Subsequently, optimal measurement positions were determined from the calculated cross-sectional dose distributions using the linear leastsquares technique. Finally, the devised $\mathrm{f}(0)^{\mathrm{CB}}{ }_{\mathrm{w}}$ method was compared to the $\mathrm{CTDI}^{\mathrm{C}}{ }_{\mathrm{w}}$ and $\mathrm{CTDI}^{\mathrm{H}}{ }_{\mathrm{w}}$ approaches.

\section{Materials and methods}

\subsection{CBCT scanning protocols}

Simulations were performed to model the CBCT protocols integrated into a Philips Allura Xper FD20 angiography scanner (Philips Healthcare, Amsterdam, Neth), a Canon Infinix Celeve-I INFX-8000 V angiography scanner (Canon Medical Systems, Tochigi, Japan), and a Varian TrueBeam linear accelerator (Varian Medical Systems, Palo Alto, CA).

The utilized scanning parameters are listed in Table 1 . The C-arm was placed on the lateral side of the patient table during the termed "roll" scan and on its head side in other cases. The rotation angle indicated the position of the X-ray tube, which could assume the following values: $0^{\circ}$ (120'clock), $90^{\circ}$ (3o'clock), $180^{\circ}$ (6o'clock), and $270^{\circ}$ (9o'clock). The tube voltages ranged from 81 to $125 \mathrm{kV}$. Beam widths were measured at the source-to-isocenter distance using a $0.6-\mathrm{cm}^{3}$ thimble ionization chamber (Model 10X5-0.6CT, Radcal Corporation, Monrovia, CA) according to the method developed by McMillan [14]. The air kerma values obtained by the ionization chamber were calibrated at the Japan Quality Assurance Organization laboratory with an uncertainty of less than $5 \%$. The TrueBeam linear accelerator was equipped with a bow-tie filter.

\subsection{Monte Carlo simulations}

\subsubsection{Validation procedure}

In this study, Electron Gamma Shower version 5 (EGS5) software was 


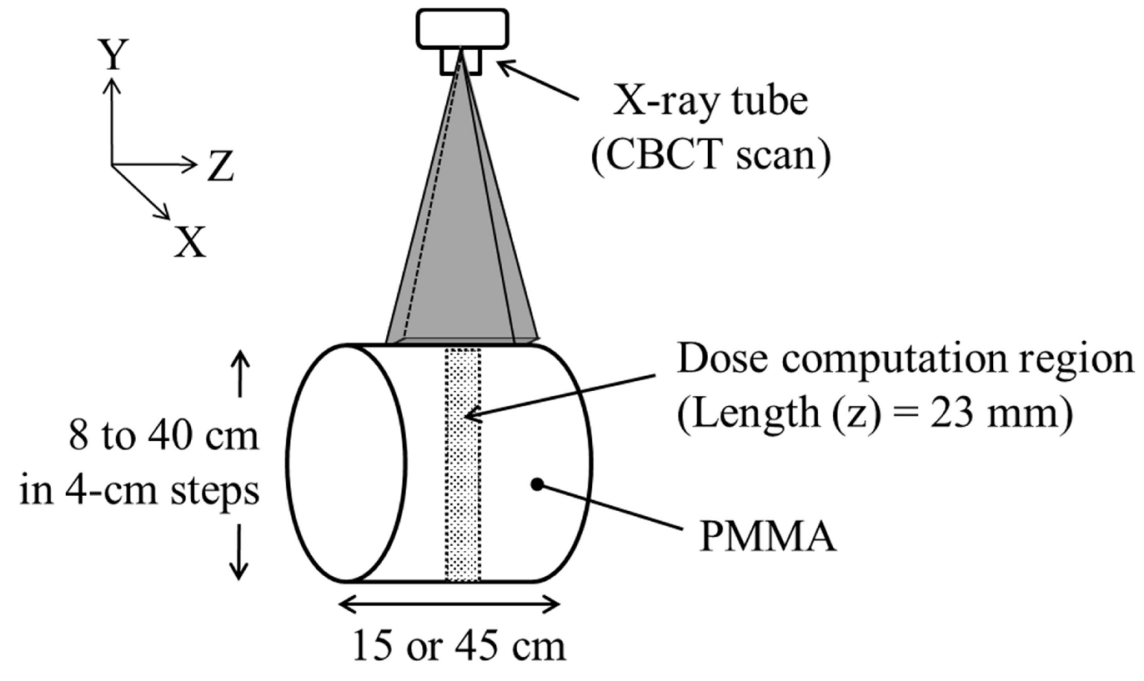

(a)

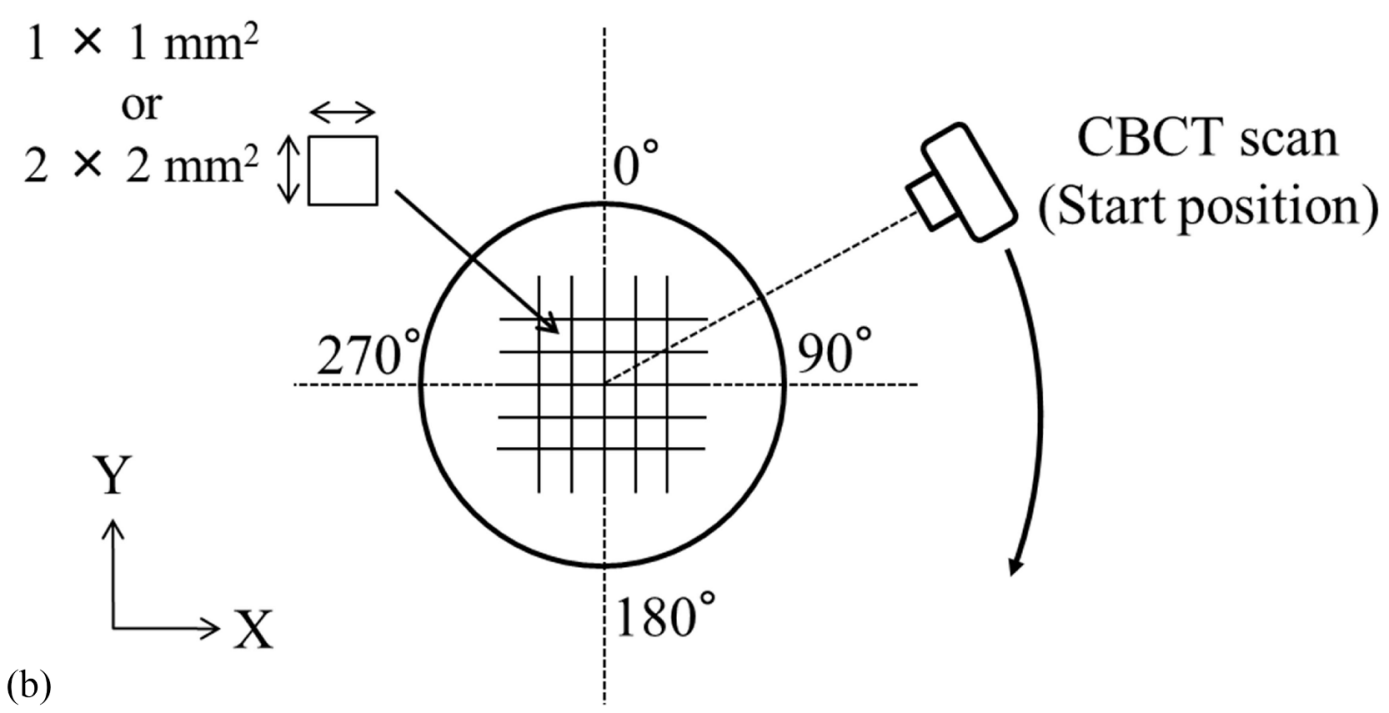

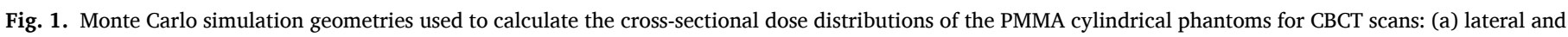
(b) cross-sectional views. The distributions were obtained by calculating the energy deposition of each voxel.

used as a Monte Carlo simulation engine, which considered photoelectric, Compton scattering, and Rayleigh scattering interactions. The original EGS5 source code was modified to conform to the CBCT scan specifications listed in Table 1. To incorporate the energy spectra of CBCT scans, equivalent sources were generated based on Tucker's formula [15] and aluminum half-value layers (HVLs) measured by a semiconductor sensor (Model AGMS-DM+, Radcal Corporation, Monrovia, CA) at the central ray. The obtained equivalent sources were similar to that reported by Turner et al. [16]. As the TrueBeam linear accelerator was equipped with a bow-tie filter, bow-tie profile measurements were also performed by using the ionization chamber. The gantry was parked at the 120 'clock position. Polystyrene foam of $15-\mathrm{cm}$ height was placed on the patient table. The ionization chamber was placed on the foam and centered at the scanner isocenter. Exposure measurements were performed by moving the table in order to obtain the bow-tie profile. Note that only the energy spectrum at the central ray was incorporated in the simulation. The cutoff energy of photons and electrons was set to $1 \mathrm{keV}$. In order to achieve statistical errors less than $1 \%$, sufficiently large numbers of photons were tallied for each simulation.

To verify the simulation accuracy, simulated CTDI values were compared to the experimentally measured values. Measurements were performed using the $0.6-\mathrm{cm}^{3}$ thimble ionization chamber and two (head and body) CTDI phantoms with a length of $15 \mathrm{~cm}$. The head CTDI phantom had a diameter of $16 \mathrm{~cm}$, whereas the diameter of the body CTDI phantom was $32 \mathrm{~cm}$. The $C T D I_{\text {center }}$ and $C T D I_{\text {periphery values were }}$ measured by implementing the CBCT scan protocols listed in Table 1 . $C T D I_{\text {periphery }}$ was calculated by averaging the results of four measurements conducted at the peripheral positions (corresponding to $90^{\circ}$ intervals). Each measurement was performed three times to reduce random error. Note that the $C T D I_{\text {center }}$ and $C T D I_{\text {periphery }}$ magnitudes were determined not by the $\mathrm{CTDI}_{100}$ method, but by the "point dose" technique.

In the Monte Carlo simulations, the simulation geometries were identical to the above-mentioned experimental geometries, and the simulated $C T D I_{\text {center }}$ and $C T D I_{\text {periphery }}$ values were obtained for each CBCT scan protocol listed in Table 1. Because the simulated doses were determined as relative values in the EGS5 software package, comparisons between the results of measurements and simulations were performed by calculating the ratios of $C T D I_{\text {periphery }}$ to $C T D I_{\text {center }}\left(R_{\mathrm{p} / \mathrm{c}}\right)$ for all CBCT scan protocols. A relative error was computed for each pair of the measured and simulated $R_{\mathrm{p} / \mathrm{c}}$ parameters to quantify the accuracy of the simulations performed in this study. 


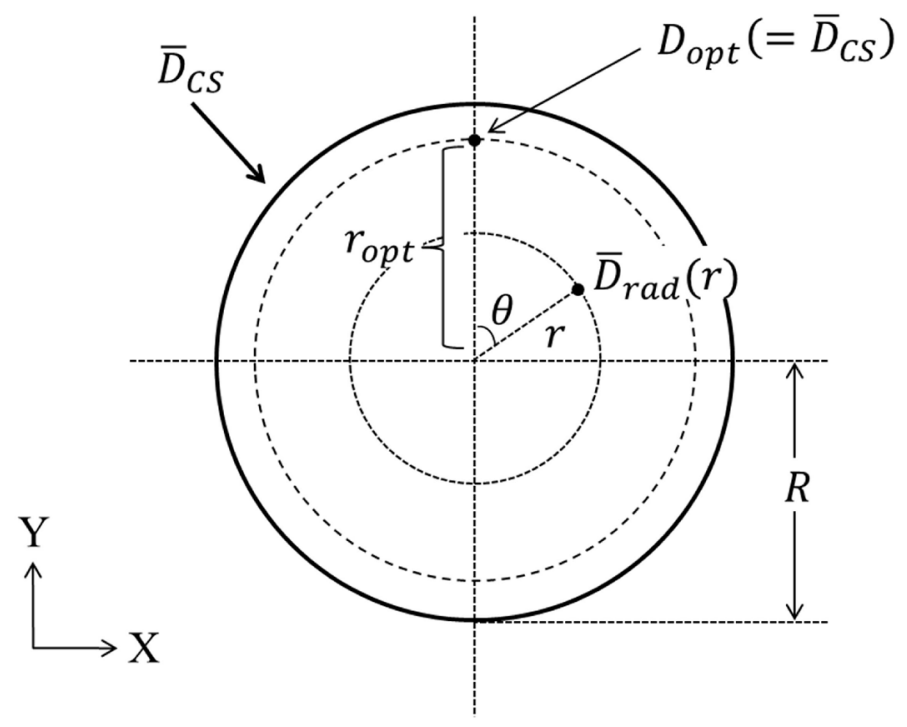

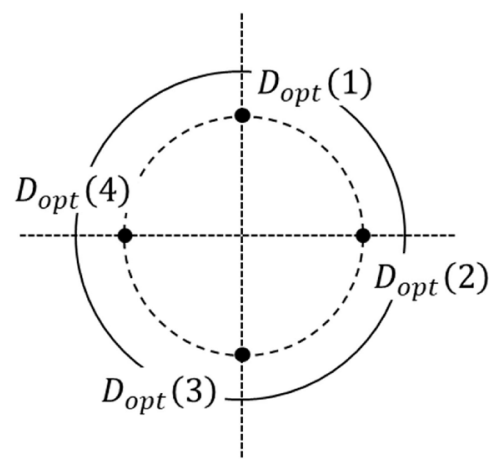

$$
I=90^{\circ}(N=4)
$$

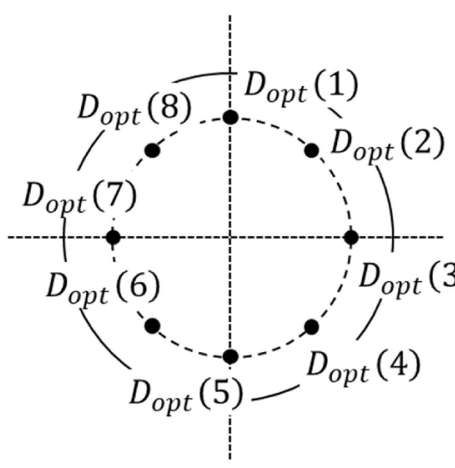

$I=45^{\circ}(N=8)$

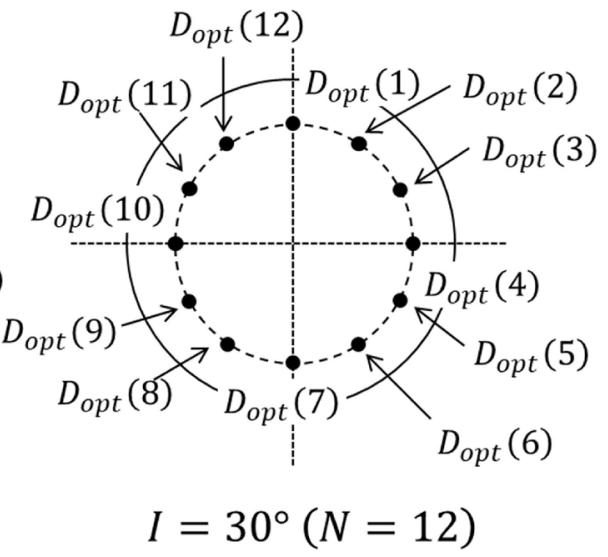

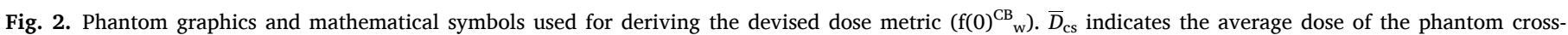

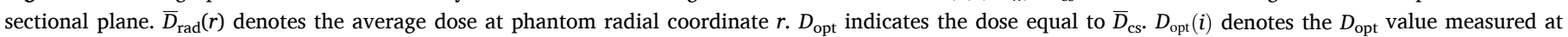
the $i$ th position of measurement interval $I$.

\subsubsection{Simulations of the cross-sectional dose distributions of PMMA cylindrical phantoms}

Monte Carlo simulations were also performed to calculate the crosssectional dose distributions of the PMMA cylindrical phantoms for CBCT scans. The resulting simulation geometries are shown in Fig. 1. The phantom models simulated in this study were represented by the PMMA cylinders with lengths of 15 and $45 \mathrm{~cm}$. The diameters of these phantoms were adjusted from 8 to $40 \mathrm{~cm}$ in $4-\mathrm{cm}$ steps. The $15-\mathrm{cm}$-long phantom was based on the CTDI 100 method, whereas the $45-\mathrm{cm}$-long phantom was based on the AAPM task group 111 method. The $\mathrm{x}, \mathrm{y}$, and $\mathrm{z}$ coordinates in this figure were defined as follows: the $\mathrm{x}-\mathrm{y}$-coordinate plane and z-coordinate axis were parallel to the cross-sectional and longitudinal axes of the cylindrical phantom, respectively. The x-coordinate axis was parallel to the floor, whereas the y-coordinate axis was perpendicular to the floor. Voxelized cylindrical phantoms were designed using the EGS5 code. Voxel sizes $(\mathrm{x} \times \mathrm{y})$ were set to $1 \times 1 \mathrm{~mm}^{2}$ for the phantoms with diameters ranging from 8 to $20 \mathrm{~cm}$ and to $2 \times 2$ $\mathrm{mm}^{2}$ for the phantoms with diameters ranging from 24 to $40 \mathrm{~cm}$. These sizes $(\mathrm{x} \times \mathrm{y})$ were small enough to calculate accurate doses because the voxel sizes used in a previous study by Abuhaimed et al. were $5 \times 5 \mathrm{~mm}^{2}$ [6]. The voxel sizes in the z-direction were set to 15 and $45 \mathrm{~cm}$ to model two PMMA cylindrical phantoms. The dose-computation region of the $\mathrm{z}$ direction with a length of $23 \mathrm{~mm}$ at the phantom center was selected to model the $0.6-\mathrm{cm}^{3}$ thimble ionization chamber. Then, we calculated the cross-sectional dose distributions with voxel sizes $(\mathrm{x} \times \mathrm{y} \times \mathrm{z})$ equal to (1 $\left.\times 1 \times 23 \mathrm{~mm}^{3}\right)$ or $\left(2 \times 2 \times 23 \mathrm{~mm}^{3}\right)$ for each CBCT scan protocol described in Table 1 and phantom model (length and diameter).

\subsection{Determination of the devised $f(0){ }^{C B}{ }_{w}$ metric for $C B C T$ dosimetry}

Fig. 2 shows the phantom graphics and mathematical symbols used for deriving the devised dose metric $\left(\mathrm{f}(0)^{\mathrm{CB}}{ }_{\mathrm{w}}\right)$. An average dose $\bar{D}_{\mathrm{cs}}$ in the phantom cross-sectional plane was calculated from the obtained crosssectional dose distributions. The value of $\bar{D}_{\mathrm{cs}}$ is defined by the expression

$\bar{D}_{\mathrm{CS}}=\frac{1}{\pi R^{2}} \int_{0}^{R} \int_{0}^{2 \pi} D(\theta, r) \mathrm{d} \theta \mathrm{d} r$

where $R$ is the cylindrical phantom radius, and $D(\theta, r)$ is the dose at angular coordinate $\theta$ and radial coordinate $r . \bar{D}_{\text {cs }}$ serves as an index for the accurate dose assessment.

An average dose $\bar{D}_{\text {rad }}(r)$ at radial coordinate $r$ was also calculated from the obtained cross-sectional dose distributions via the formula

$\bar{D}_{\text {rad }}(r)=\frac{1}{2 \pi r} \int_{0}^{2 \pi} D(\theta, r) \mathrm{d} \theta$

The magnitudes of $\bar{D}_{\text {cs }}$ and $\bar{D}_{\text {rad }}(r)$ were computed for all CBCT scan protocols listed in Table 1. By performing a linear least squares fit of the 
Table 2

Measured and simulated ratios $\left(R_{\mathrm{p} / \mathrm{c}}\right)$ between the CTDI periphery and CTDI $I_{\text {center }}$ values obtained for various CBCT scan protocols. Measurements of the $C T D I_{\text {per- }}$ iphery and $C T D I_{\text {center }}$ parameters were performed using the $0.6-\mathrm{cm}^{3}$ thimble ionization chamber and standard head and body CTDI phantoms.

\begin{tabular}{|c|c|c|c|c|c|}
\hline \multirow{2}{*}{$\begin{array}{l}\text { Scan } \\
\text { region }\end{array}$} & \multirow{2}{*}{$\begin{array}{l}\text { Scanner } \\
\text { model }\end{array}$} & \multirow{2}{*}{$\begin{array}{l}\text { Termed scan } \\
\text { protocol }\end{array}$} & \multicolumn{2}{|l|}{$R_{\mathrm{p} / \mathrm{c}}$} & \multirow{2}{*}{$\begin{array}{l}\text { Relative } \\
\text { error [\%] }\end{array}$} \\
\hline & & & $\begin{array}{l}\text { Measured } \\
( \pm \mathrm{SD})\end{array}$ & $\begin{array}{l}\text { Simulated } \\
( \pm \text { SD })\end{array}$ & \\
\hline \multirow[t]{6}{*}{ Head } & \multirow[t]{3}{*}{$\begin{array}{l}\text { Allura Xper } \\
\text { FD20 }\end{array}$} & Head-prop & $\begin{array}{l}1.33 \\
( \pm 0.02)\end{array}$ & $\begin{array}{l}1.31 \\
( \pm 0.01)\end{array}$ & -1.5 \\
\hline & & Head-roll & $\begin{array}{l}1.23 \\
( \pm 0.01)\end{array}$ & $\begin{array}{l}1.30 \\
( \pm 0.01)\end{array}$ & 5.7 \\
\hline & & $\begin{array}{l}\text { Head- } \\
\text { XperCT }\end{array}$ & $\begin{array}{l}1.06 \\
( \pm 0.01)\end{array}$ & $\begin{array}{l}1.15 \\
( \pm 0.01)\end{array}$ & 8.5 \\
\hline & \multirow[t]{2}{*}{$\begin{array}{l}\text { Infinix } \\
\text { Celeve-I }\end{array}$} & Head-3D & $\begin{array}{l}1.37 \\
( \pm 0.01)\end{array}$ & $\begin{array}{l}1.27 \\
( \pm 0.01)\end{array}$ & -7.3 \\
\hline & & $\begin{array}{l}\text { Head- } \\
\text { AlphaCT }\end{array}$ & $\begin{array}{l}1.28 \\
( \pm 0.01)\end{array}$ & $\begin{array}{l}1.31 \\
( \pm 0.01)\end{array}$ & 2.3 \\
\hline & TrueBeam & Head & $\begin{array}{l}0.93 \\
( \pm 0.01)\end{array}$ & $\begin{array}{l}0.99 \\
( \pm 0.01)\end{array}$ & 6.5 \\
\hline \multirow[t]{4}{*}{ Body } & \multirow[t]{3}{*}{$\begin{array}{l}\text { Allura Xper } \\
\text { FD20 }\end{array}$} & Body-prop & $\begin{array}{l}2.56 \\
( \pm 0.01)\end{array}$ & $\begin{array}{l}2.58 \\
( \pm 0.02)\end{array}$ & 0.8 \\
\hline & & Body-roll & $\begin{array}{l}2.30 \\
( \pm 0.02)\end{array}$ & $\begin{array}{l}2.37 \\
( \pm 0.02)\end{array}$ & 3.0 \\
\hline & & $\begin{array}{l}\text { Body- } \\
\text { XperCT }\end{array}$ & $\begin{array}{l}1.84 \\
( \pm 0.01)\end{array}$ & $\begin{array}{l}1.90 \\
( \pm 0.01)\end{array}$ & 3.3 \\
\hline & TrueBeam & $\begin{array}{l}\text { Body- } \\
\text { spotlight } \\
\text { (Fan type: } \\
\text { full) }\end{array}$ & $\begin{array}{l}1.63 \\
( \pm 0.01)\end{array}$ & $\begin{array}{l}1.59 \\
( \pm 0.01)\end{array}$ & -2.5 \\
\hline
\end{tabular}

full set of the $\bar{D}_{\text {cs }}$ and $\bar{D}_{\text {rad }}(r)$ parameters, we determined the optimal radial coordinate $r_{\text {opt }}$, which represented a measurement position for the average phantom dose $D_{\text {opt }}$.

Subsequently, an adequate measurement interval $I$ on the phantom circumference corresponding to $r_{\text {opt }}$ was obtained by conducting several measurements. The values of $I$ were determined at angles of $90^{\circ}, 45^{\circ}$, and $30^{\circ}$ (corresponding to the measurement position numbers $N=4,8$, and 12, respectively). The angular definition of $I$ is provided in Fig. 1(b). For example, at $I=90^{\circ}$, the measurement positions were 3, 6, 9, and 120 'clock. We calculated the dose $\bar{D}_{\text {opt }}^{\mathrm{int}=I}$ averaged over the values of $D_{\mathrm{opt}}$ at the measurement positions of interval $I$ in the form

$\bar{D}_{\mathrm{opt}}^{\mathrm{int}=I}=\frac{\sum_{i=1}^{N} \mathrm{D}_{\mathrm{opt}}(i)}{N}$

The values of $\bar{D}_{\mathrm{opt}}^{\mathrm{int}=90^{\circ}}, \bar{D}_{\mathrm{opt}}^{\mathrm{int}=45^{\circ}}$, and $\bar{D}_{\mathrm{opt}}^{\mathrm{int}=30^{\circ}}$ obtained via Eq. (6) were compared with $\bar{D}_{\mathrm{cs}}$, and a suitable measurement interval $I$ was determined. Finally, the devised $\mathrm{f}(0)^{\mathrm{CB}}{ }_{\mathrm{w}}$ metric was defined as

$f(0)_{w}^{C B}=\frac{\sum_{i=1}^{N} \mathrm{D}_{\mathrm{opt}}(i)}{N}$

where $N$ is the number of measurement positions obtained from the corresponding measurement interval $I$. The values of $r_{\text {opt }}, I$, and $f(0){ }^{C B}{ }_{w}$ were calculated for all phantom diameters $(d=8-40 \mathrm{~cm})$ and lengths $(L$ $=15$ and $45 \mathrm{~cm}$ ) using the above-mentioned procedure.

\section{4. $f(0){ }^{C B}{ }_{w}$ validity assessment}

To ascertain the validity of the devised $\mathrm{f}(0){ }^{\mathrm{CB}}{ }_{\mathrm{w}}$ metric, its values were compared with the $C T D I^{C}{ }_{w}$ and $C T D I^{H}{ }_{w}$ magnitudes calculated via Eqs. (1) and (3) and simulated cross-sectional dose distributions of the PMMA cylindrical phantom. Note that the $C T D I_{w}$ values were computed by the "point dose" method proposed in the AAPM task group 111 report. The relative difference $P D$ between $\bar{D}_{\text {cs }}$ and each dose value $D_{\mathrm{w}}$ was evaluated for each phantom diameter, length, and CBCT scan protocol as follows:
[Relative dose] High

(a)
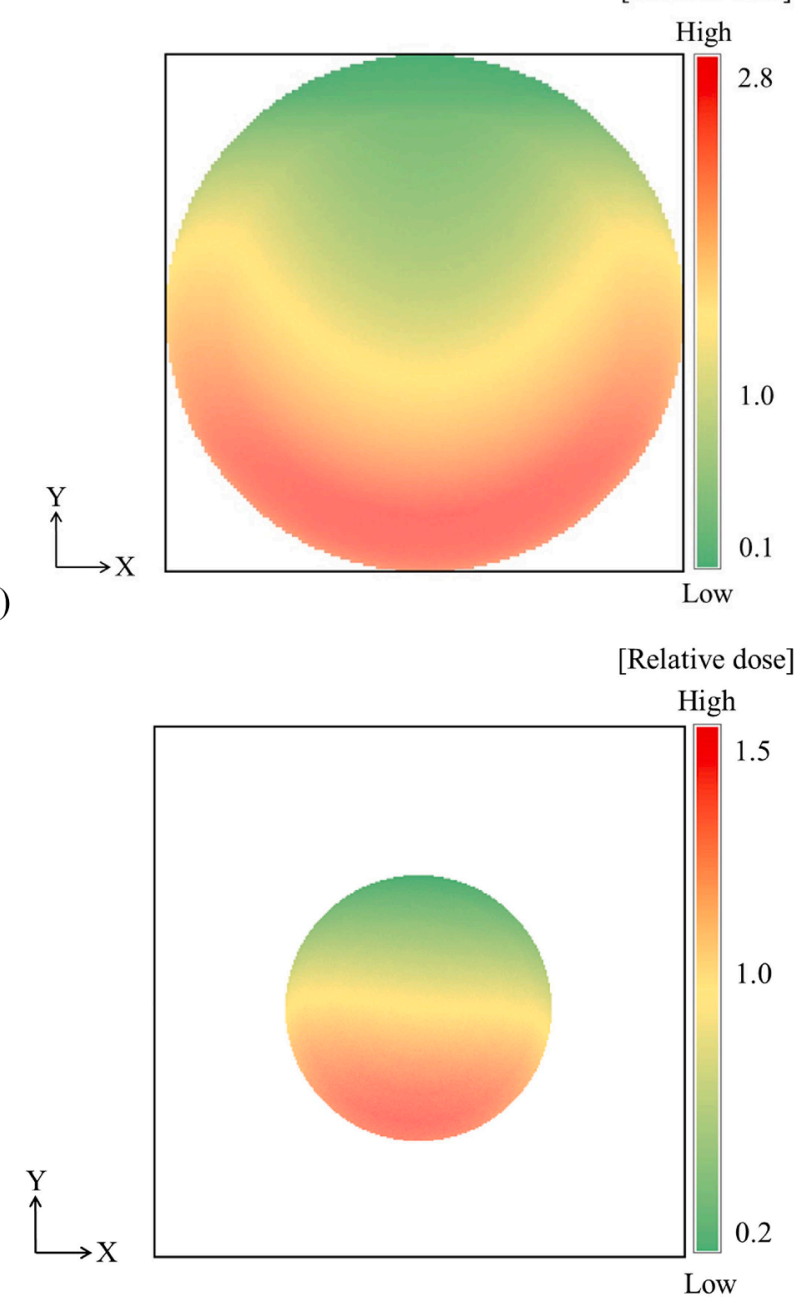

(b)

Fig. 3. Visualizations of the simulated cross-sectional dose distributions for the (a) body phantom $(d=32 \mathrm{~cm}$ and $L=45 \mathrm{~cm})$ and "Allura Xper FD20 - BodyXperCT" scan protocol, and (b) head phantom $(d=16 \mathrm{~cm}$ and $L=45 \mathrm{~cm})$ and "TrueBeam - Head" scan protocol. The relative doses are normalized with respect to the values obtained for the central positions.

$P D=\frac{D_{\mathrm{w}}-\bar{D}_{\mathrm{CS}}}{\bar{D}_{\mathrm{CS}}} \times 100$

where $D_{\mathrm{w}}$ indicates $f(0)^{C B}{ }_{w}, C T D I^{C}{ }_{w}$, or $C T D I^{H}{ }_{w}$.

\section{Results}

Table 2 lists the relative errors calculated for all pairs of the measured and simulated $R_{\mathrm{p} / \mathrm{c}}$ values. The maximum errors of $8.5 \%$ and $3.3 \%$ were obtained for the head and body scan protocols, respectively, indicating a good agreement between these two datasets.

Fig. 3 shows the visualizations of the simulated cross-sectional dose distributions of the (a) phantom with $d=32 \mathrm{~cm}$ and $L=45 \mathrm{~cm}$ ("Allura Xper FD20 - Body-XperCT" scan protocol), and (b) phantom with $d=16$ $\mathrm{cm}$ and $L=45 \mathrm{~cm}$ ("TrueBeam - Head" scan protocol). The dose value at each phantom position was normalized with respect to the central dose. The doses at the irradiated angles were larger than those at the nonirradiated angles.

The dose profiles of $\bar{D}_{\text {rad }}(r)$ are shown in Fig. 4. The $\bar{D}_{\text {cs }}$ values indicated by the filled circles are plotted on each $\bar{D}_{\text {rad }}(r)$ profile. The 

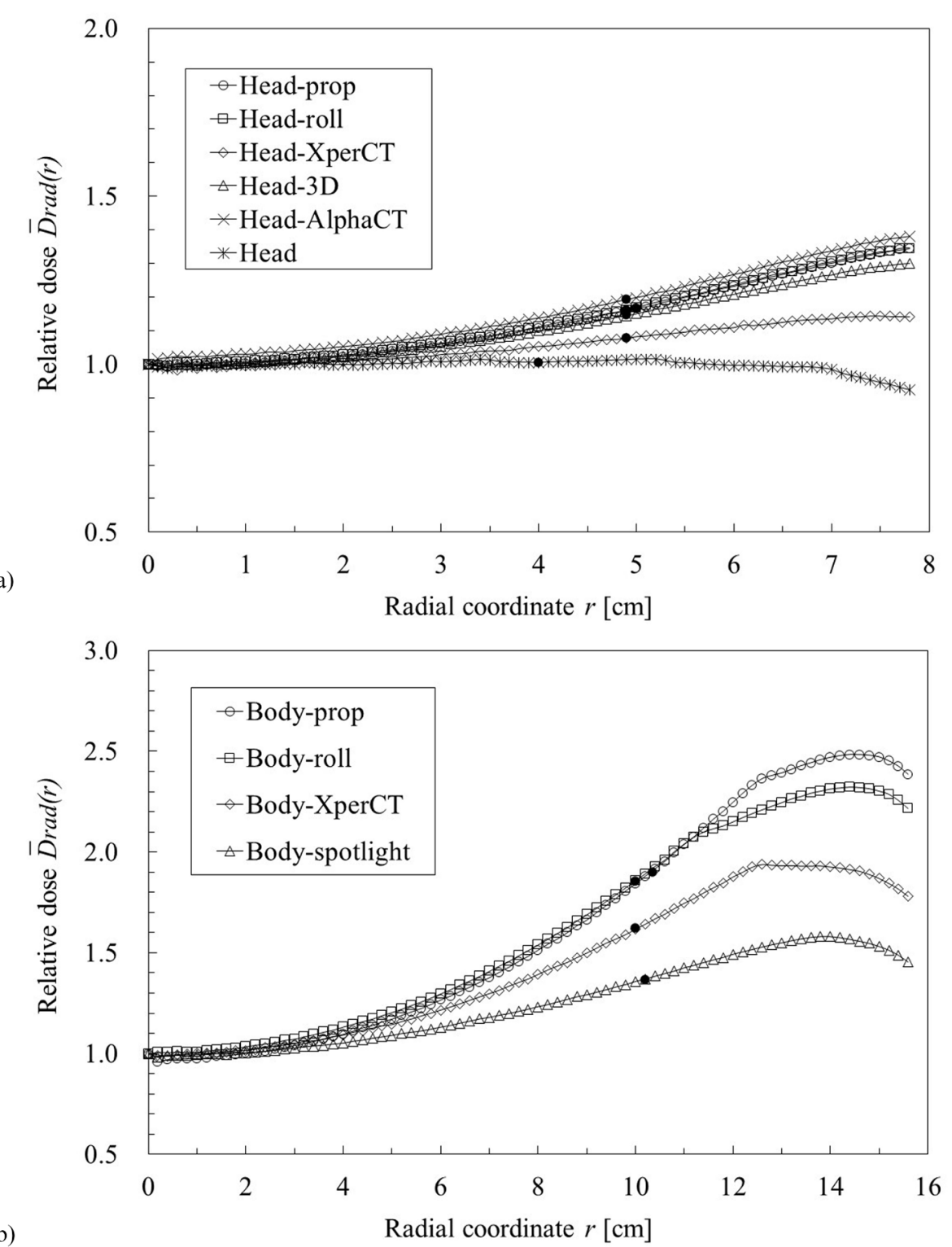

Fig. 4. $\bar{D}_{\text {rad }}(r)$ profiles of the (a) head phantom $(d=$ $16 \mathrm{~cm}$ and $L=15 \mathrm{~cm})$ and (b) body phantom $(d=32$ $\mathrm{cm}$ and $L=15 \mathrm{~cm}$ ) recorded for various CBCT scan protocols. $\bar{D}_{\text {rad }}(r)$ profiles obtained at (c) various phantom diameters $(d=8,16,32$, and $40 \mathrm{~cm})$ and (d) two phantom lengths $(L=15$ and $45 \mathrm{~cm}$ ) for the propeller scan protocols ("Head-prop" and "Bodyprop") of the Allura Xper FD20 scanner. The termed scan protocols in panels (a-d) are identical to those listed in Table 1 . The $\bar{D}_{\text {rad }}(r)$ values were normalized with respect to the central dose $\left(\bar{D}_{\text {rad }}(0)\right)$ for each profile. The $\bar{D}_{\text {cs }}$ values are indicated by the filled circles $(a, b, d)$.

(b)

$\bar{D}_{\text {rad }}(r)$ values were normalized with respect to the central dose $\left(\bar{D}_{\text {rad }}(0)\right)$ for all profiles. Fig. 4 (a) shows the $\bar{D}_{\text {rad }}(r)$ profiles of the head phantom ( $d=16 \mathrm{~cm}$ and $L=15 \mathrm{~cm}$ ) obtained for various CBCT scan protocols, whereas Fig. 4(b) displays those of the body phantom $(d=32 \mathrm{~cm}$ and $L$ $=15 \mathrm{~cm}$ ). The dose profiles of the head phantom are almost linear, while those of the body phantom first gradually increase and then decrease with increasing radial coordinate $r$ from the phantom center. This characteristic of the body phantom's dose profile is called a "gull wing shape" by Bakalyar et al. [17]. Furthermore, the dose increments for the linear accelerator are more gradual than those for the angiography scanners. Fig. 4(c) compares the $\bar{D}_{\text {rad }}(r)$ profiles of the phantoms with various diameters obtained for the "Head-prop" and "Body-prop" scan protocols of the Allura Xper FD20 scanner. Here, the "gull wing shape" effect becomes stronger with increasing phantom diameter. The $\bar{D}_{\text {rad }}(r)$ profiles obtained for the phantoms with lengths of 15 and $45 \mathrm{~cm}$, diameters of 16 and $32 \mathrm{~cm}$, and the "Head-prop" and "Body-prop" scan protocols of the Allura Xper FD20 scanner are shown in Fig. 4(d). The radial coordinates corresponding to the $\bar{D}_{\mathrm{cs}}$ values show good agreement between the phantoms with lengths of 15 and $45 \mathrm{~cm}$ at both diameters $(d=16$ and $32 \mathrm{~cm})$.
The optimal radial coordinates $r_{\text {opt }}$ corresponding to the average doses $D_{\text {opt }}$ determined by the linear least-squares technique are listed in Table 3 and Fig. 5. Very good agreement is observed between the $r_{\mathrm{opt}}$ values obtained at phantom lengths of 15 and $45 \mathrm{~cm}$. Furthermore, $r_{\mathrm{opt}}$ is linearly proportional to the phantom diameter (Fig. 5).

Fig. 6 shows the differences between the $\bar{D}_{\text {opt }}^{\mathrm{int}=I}\left(\bar{D}_{\mathrm{opt}}^{\mathrm{int}=90^{\circ}}, \bar{D}_{\mathrm{opt}}^{\mathrm{int}=45^{\circ}}\right.$, and $\bar{D}_{\text {opt }}^{\text {int=30 }}{ }^{\circ}$ ) and $\bar{D}_{\text {cs }}$ values of the 15-cm-long phantoms with diameters of (a) 16 and (b) $32 \mathrm{~cm}$ obtained for various CBCT scan protocols. Small differences were observed between the three measurement intervals $I$, although a relatively large difference of $1.7 \%$ was obtained at $I=90^{\circ}$ for the phantom with a diameter of $32 \mathrm{~cm}$ and "Body-spotlight" scan protocol of the linear accelerator. Under all simulation conditions (phantom diameters, phantom lengths, and CBCT scan protocols), maximum differences of $2.5 \%$ (mean $=-0.03 \%$ ), $-1.2 \%$ (mean $=-0.07 \%$ ), and $-1.5 \%$ (mean $=-0.29 \%$ ) were observed for $I=90^{\circ}, 45^{\circ}$, and $30^{\circ}$, respectively. These results indicate that the measurement interval $I=90^{\circ}$ (corresponding to the number of measurement positions $N=4$ ) was sufficient for estimating the average phantom dose for CBCT scans. Thus, by substituting $N=4$ in Eq. (7), the $\mathrm{f}(0){ }^{\mathrm{CB}}$ w metric was defined at the 


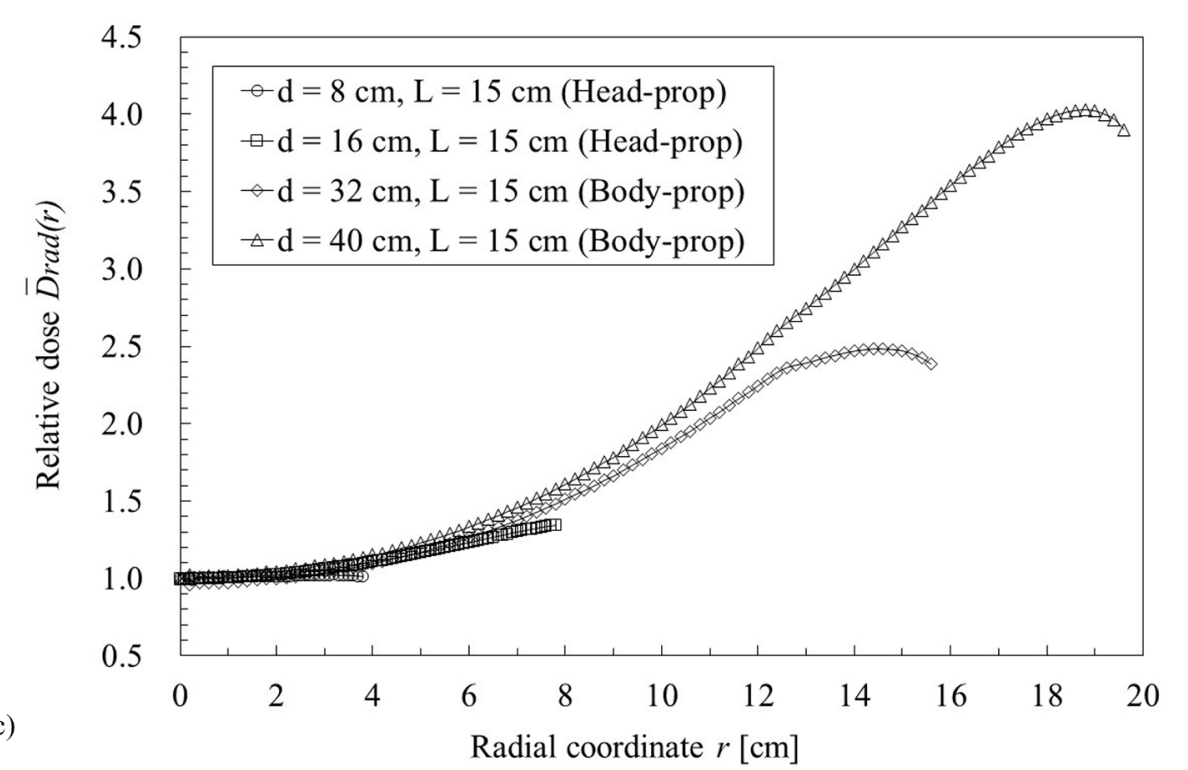

(c)

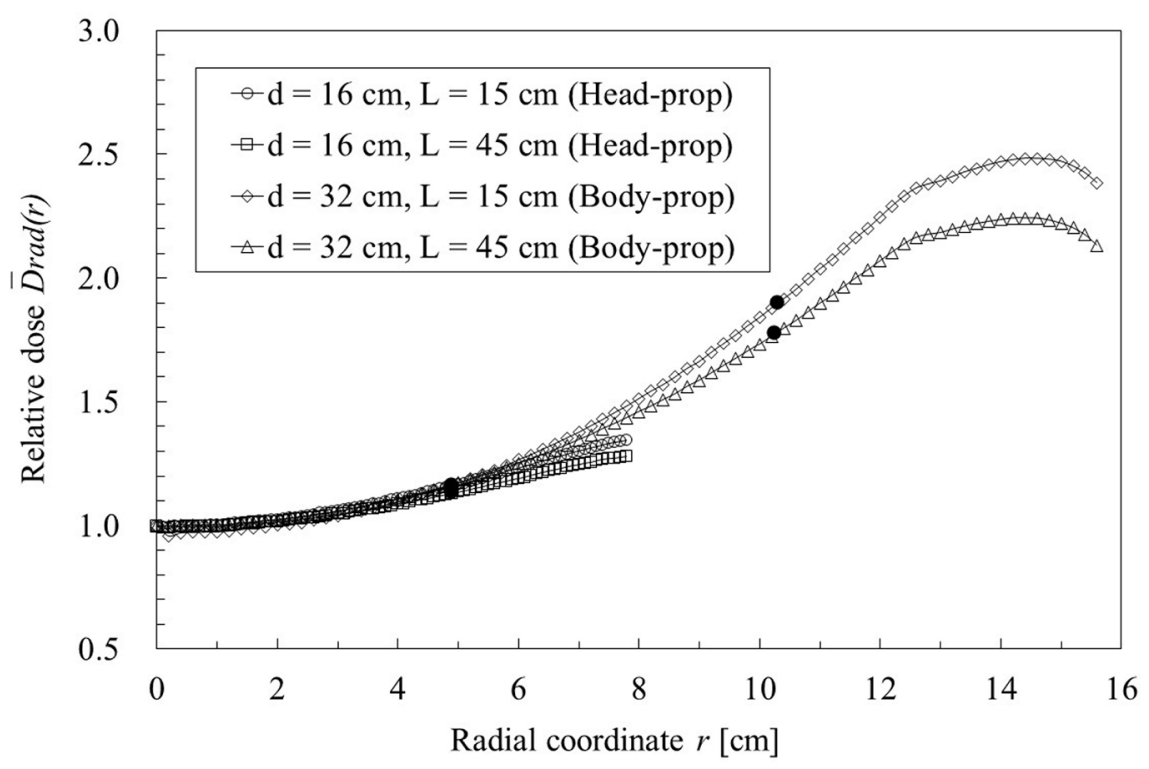

Fig. 4. (continued).

Table 3

Optimal radial coordinates $r_{\text {opt }}$ corresponding to the average doses $D_{\text {opt }}$ obtained at phantom lengths $L=15$ and $45 \mathrm{~cm}$. The $r_{\mathrm{opt}}$ values denote the distances from the phantom center.

\begin{tabular}{lcc}
\hline Phantom diameter $[\mathrm{cm}]$ & \multicolumn{2}{c}{ Optimal radial coordinate $r_{\mathrm{opt}}[\mathrm{cm}]$} \\
\cline { 2 - 3 } & $L=15 \mathrm{~cm}$ & $L=45 \mathrm{~cm}$ \\
\hline 8 & 1.9 & 1.9 \\
12 & 3.6 & 3.6 \\
16 & 4.9 & 4.9 \\
20 & 6.3 & 6.3 \\
24 & 7.8 & 7.8 \\
28 & 9.0 & 9.0 \\
32 & 10.2 & 10.0 \\
36 & 11.4 & 11.2 \\
40 & 13.0 & 12.8 \\
\hline
\end{tabular}

measurement positions depicted in Fig. 7 as

$f(0)_{w}^{C B}=\frac{\sum_{i=1}^{4} D_{\mathrm{opt}}(i)}{4}$

The relative differences between the $\bar{D}_{\mathrm{cs}}$ and each dose parameter $(f$
$(0)^{C B}{ }_{w}, C T D I^{C}{ }_{w}$, and $C T D I^{H}{ }_{w}$ values) obtained at different phantom lengths $(L=15$ and $45 \mathrm{~cm}$ ) are shown in Fig. 8. Here, each symbol denotes the relative difference value averaged for all CBCT scan protocols listed in Table 1 at a given phantom diameter, and the corresponding error bar indicates its maximum and minimum magnitudes. The maximum differences of $4.1 \%, 16.7 \%$, and $11.9 \%$ were obtained for the $\mathrm{f}(0)^{\mathrm{CB}}{ }_{\mathrm{w}}, \mathrm{CTDI}_{\mathrm{w}}^{\mathrm{C}}$, and $\mathrm{CTDI}^{\mathrm{H}}{ }_{\mathrm{w}}$ methods, respectively. In addition, the smallest variation for various CBCT scan protocols was observed when the $\mathrm{f}(0){ }_{\mathrm{w}}^{\mathrm{CB}}$ method was utilized.

\section{Discussion}

In this study, we devised a new dose metric $\left(\mathrm{f}(0)^{\mathrm{CB}}{ }_{\mathrm{w}}\right)$ for $\mathrm{CBCT}$ dosimetry to determine a more accurate average dose by performing Monte Carlo simulations and using the linear least-squares technique. The $f(0){ }^{C B}$ value can be calculated by averaging four "point doses" at $90^{\circ}$ intervals and the optimal positions $r_{\text {opt }}$ listed in Table 3.

As shown in Table 2, the Monte Carlo simulation accuracy was successfully validated in this work as the average differences between the simulated and measured $R_{\mathrm{p} / \mathrm{c}}$ values were $5.3 \%$ and $2.4 \%$ for the head and body scan regions, respectively. The maximum difference of 


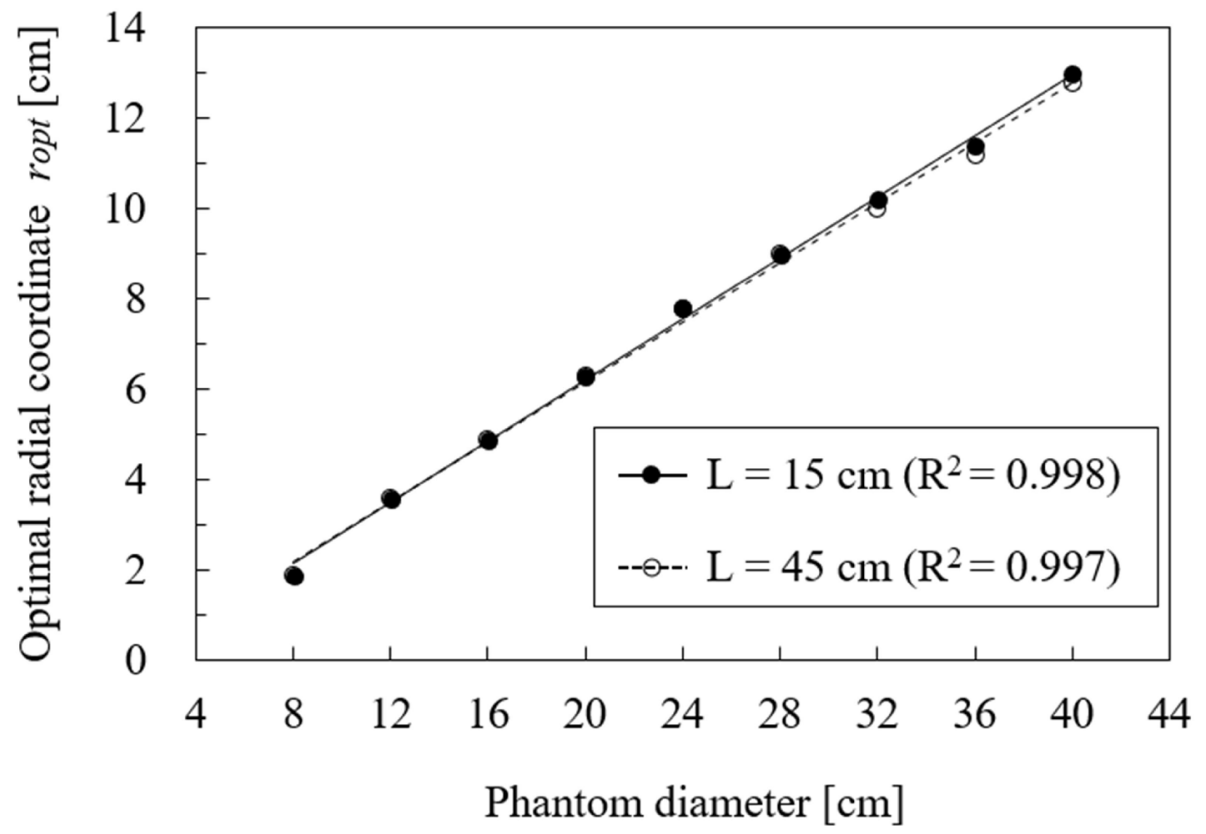

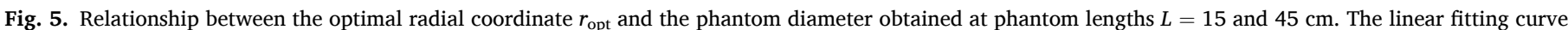
and correlation coefficient $\mathrm{R}^{2}$ are shown as well.

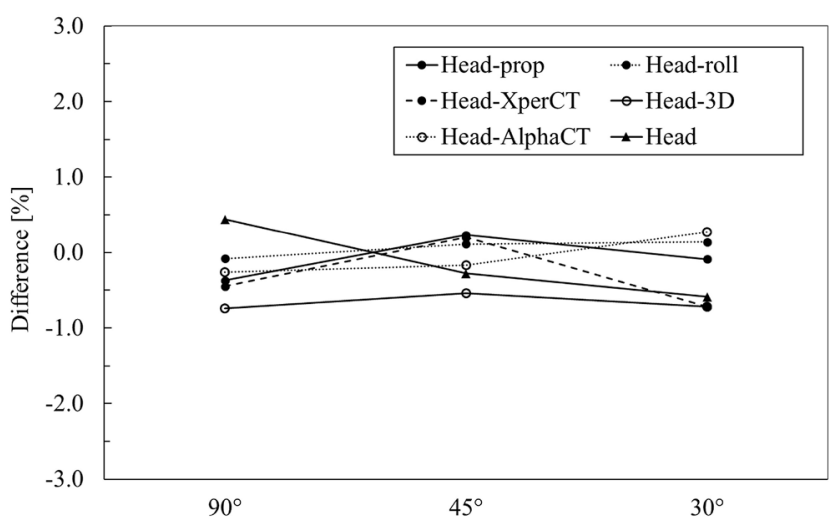

(a) Interval $I$ between measurement positions

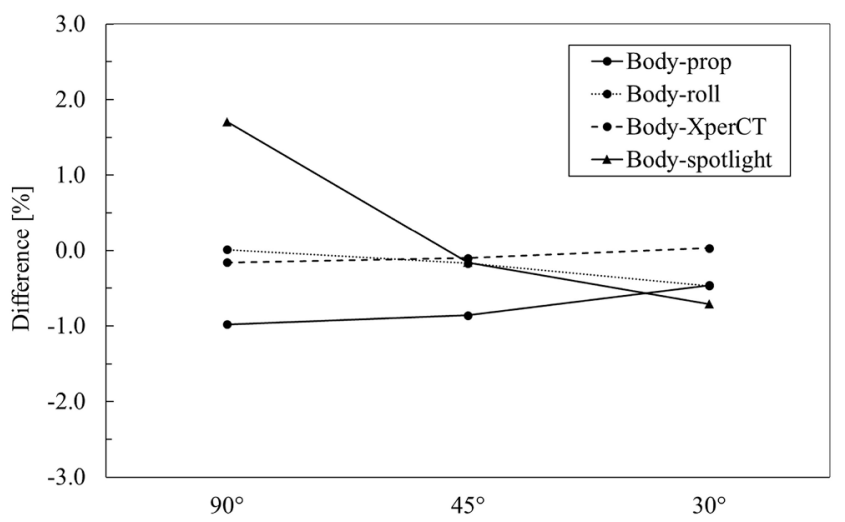

(b)

Interval $I$ between measurement positions

Fig. 6. Differences between the $\bar{D}_{\mathrm{opt}}^{\mathrm{int}=I}\left(\bar{D}_{\mathrm{opt}}^{\mathrm{int}=90^{\circ}}, \bar{D}_{\mathrm{opt}}^{\mathrm{int}=45^{\circ}}\right.$, and $\left.\bar{D}_{\mathrm{opt}}^{\mathrm{int}=30^{\circ}}\right)$ and $\bar{D}_{\mathrm{cs}}$ values obtained for the 15-cm-long phantoms with diameters of (a) 16 and (b) $32 \mathrm{~cm}$ and various CBCT scan protocols. $I$ denotes the interval between measurement positions on the phantom circumference at $r_{\text {opt }}$.
$8.5 \%$ was computed for the head scan region. The average and maximum differences obtained in this study were in good agreement with those reported in Abuhaimed's work [5]. Larger differences were observed for the head scan region because the proprietary nature of the actual material and composition of the patient table kept us from considering it in the simulations. Abuhaimed et al. concluded that the omission of the patient table might have a greater impact on the results obtained for the lower attenuation head phantom [6].

Fig. 3 shows the non-radially symmetric dose distributions owing to the partial angle irradiation for CBCT scans. Fig. 4(a, b) show that the dose increment toward the phantom surface (i.e., at larger $r$ ) is more gradual for the linear accelerator (TrueBeam) than for the angiography scanners because the former is equipped with the bow-tie filters. As the doses decreased with increasing fan-beam angles because of the increasing thickness of the bow-tie filter [18], the doses at larger $r$ decreased as well. Although the obtained dose profiles were divergent for various CBCT scan protocols and phantom lengths depicted in Fig. 4 (a, b, d), the radial coordinates corresponding to the average dose $\bar{D}_{\mathrm{cs}}$ were consistent, indicating that the optimal radial coordinate $r_{\text {opt }}$ was independent of the CBCT scan protocol (scanner type, rotation angle, and tube voltage) and phantom length ( $L=15$ and $45 \mathrm{~cm}$ ). While the radial coordinates corresponding to $\bar{D}_{\mathrm{cs}}$ for the linear accelerator were different from those obtained for the angiography scanners and head phantom (Fig. 4(a)), it would not affect the $r_{\text {opt }}$ analysis because the dose profile of the linear accelerator was almost linear. The $r_{\mathrm{opt}}$ values obtained by the linear least-squares technique (see Table 3 and Fig. 5) were independent of the phantom lengths and linearly increased with phantom diameters. These results indicate that the devised $\mathrm{f}(0)^{\mathrm{CB}}{ }_{\mathrm{w}}$ metric described by Eq. (9) and Fig. 7 is applicable to the point dose methods utilized for the phantoms with lengths of 15 and $45 \mathrm{~cm}$. Recently, the AAPM task group 200 report proposed a new phantom of polyethylene of 30-cm diameter for CT radiation dosimetry [19]. In the case where a new phantom model is proposed, the data presented in Table 3 and Fig. 5 are useful to give optimal radial positions for average dose evaluations.

By taking into account the results displayed in Fig. 6, it can be concluded that the measurement interval $I=90^{\circ}$ is adequate for estimating the average phantom dose for a CBCT scan. At $I=90^{\circ}, f(0)^{C B}{ }_{w}$ is determined by measuring doses only at the four positions depicted in 


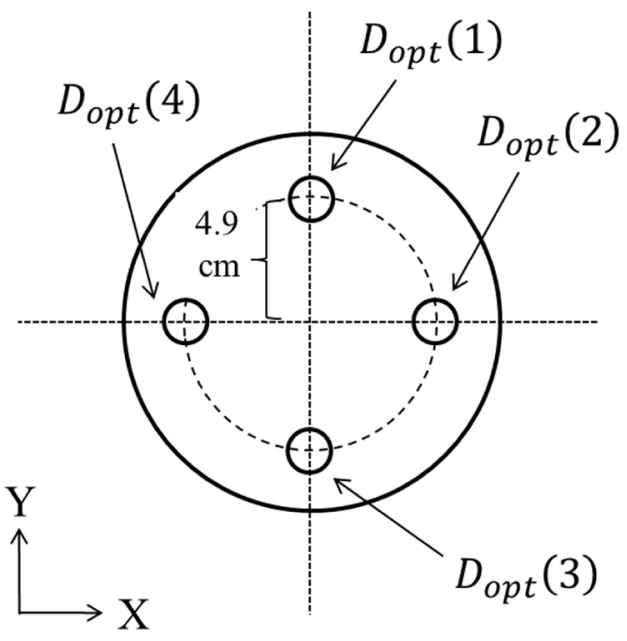

$$
\begin{gathered}
d=16 \mathrm{~cm} \\
\text { (Head phantom) }
\end{gathered}
$$

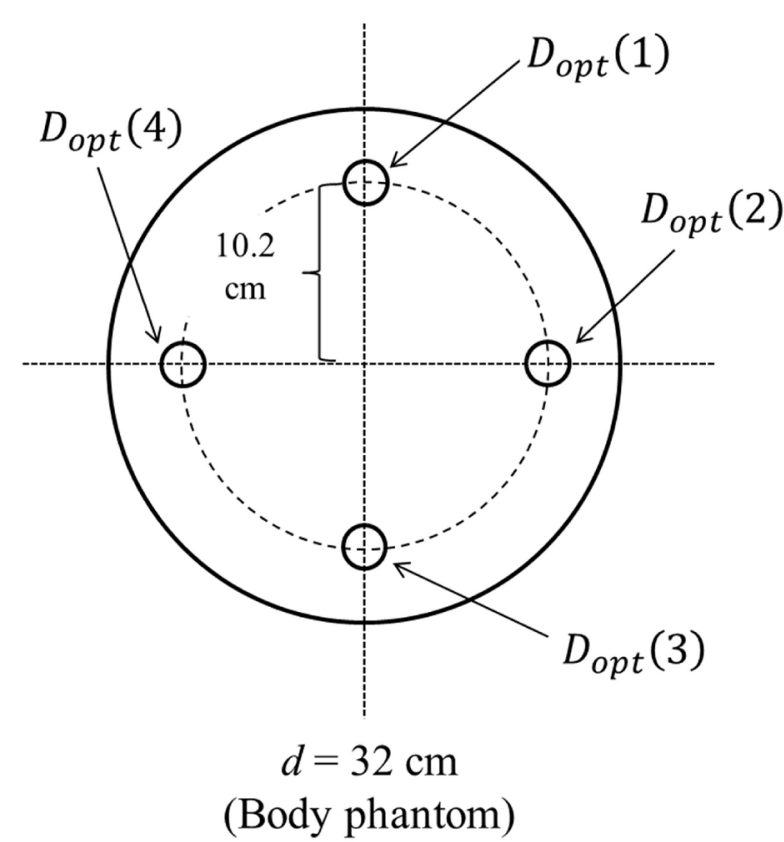

(Body phantom)

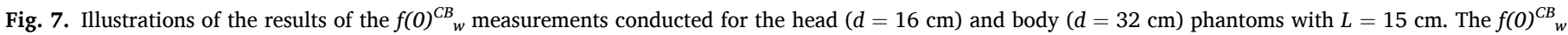

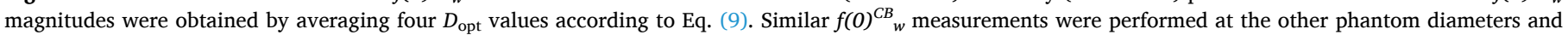
lengths using the $r_{\text {opt }}$ values listed in Table 3.

Fig. 7. Compared with the previously developed $\operatorname{CTDI}_{\mathrm{w}}\left(\mathrm{CTDI}^{\mathrm{C}}{ }_{\mathrm{w}}\right.$ and $\mathrm{CTDI}^{\mathrm{H}}{ }_{\mathrm{w}}$ ) methods, which are required to measure doses at five (one central and four peripheral) positions in the CTDI phantom, the devised $f$ $(0)^{\mathrm{CB}}{ }_{\mathrm{w}}$ method is time-saving and more comfortable in terms of the heat generated by the X-ray equipment. Although our devised method needs to modify the CTDI phantoms, the effort needed to drill standard CTDI phantoms, which are in widespread use, at optimal radial positions is not significant.

As shown in Fig. 8, the devised $\mathrm{f}(0)^{\mathrm{CB}}{ }_{\mathrm{w}}$ method provides the best fit for the average dose among various dose metrics. The $\mathrm{f}(0){ }^{\mathrm{CB}}{ }_{\mathrm{w}}$ metric is independent of the phantom diameter, whereas the relative difference values obtained by the $\mathrm{CTDI}^{\mathrm{C}}{ }_{\mathrm{w}}$ and $\mathrm{CTDI}^{\mathrm{H}}{ }_{\mathrm{w}}$ methods vary with phantom diameters (this trend was more pronounced at large diameters). The AAPM task group 204 report has developed a dose evaluation index named SSDE to consider the effect of patient size in the X-ray CT dose evaluation $[13,20,21]$. Size correction factors to calculate SSDE were derived based on the $\mathrm{CTDI}^{\mathrm{C}}{ }_{\mathrm{w}}$ method for a wide range of phantom diameters. The results of Fig. 8 were obtained for a wide range of phantom diameters and would be useful to adapt CBCT dose values to SSDE. Note that size correction factors in the AAPM task group 204 report were derived for water or tissue-equivalent materials, whereas the results of Fig. 8 were derived for PMMA phantoms. Therefore, in the near future, we plan to simulate cross-sectional dose distributions of water phantoms to propose size correction factors for the $\mathrm{f}(0)^{\mathrm{CB}}{ }_{\mathrm{w}}$ method. In addition, the $\mathrm{f}(0)^{\mathrm{CB}}{ }_{\mathrm{w}}$ method is virtually independent of the CBCT scanner type and utilized protocol owing to the smallest error bar range among various dose metrics. These results indicate that the devised $\mathrm{f}(0)^{\mathrm{CB}}{ }_{\mathrm{w}}$ metric is appropriate for the CBCT dose evaluations with partial angle irradiations. Although the $C T D I_{w}\left(C T D I^{C}{ }_{w}\right.$ and $\left.C T D I^{H}{ }_{w}\right)$ values $d=20,24,36$, and $40 \mathrm{~cm}$ are divergent to average doses, the values $d=16$ and $32 \mathrm{~cm}$ (standard head and body CTDI phantom diameters) correspond fortuitously to average doses. Therefore, the previous $\mathrm{CTDI}_{\mathrm{w}}\left(\mathrm{CTDI}^{\mathrm{C}}{ }_{\mathrm{w}}\right.$ and $\mathrm{CTDI}^{\mathrm{H}}{ }_{\mathrm{w}}$ ) methods would still be convenient for routine quality control purposes when it is difficult to equip our devised phantoms.

In terms of the dose estimation, the long phantom $(L=45 \mathrm{~cm})$ is preferable to the short phantom $(L=15 \mathrm{~cm})$, owing to the large longitudinal beam width of the scattering radiation components utilized for CBCT scanning. However, the long phantom is relatively heavy, and it may be difficult for medical physicists to use it in a quality assurance procedure. Therefore, in several studies, the long phantom dose was estimated from the short phantom dose using correction factors (shortphantom to long-phantom dose ratios) $[6,8]$. A similar relationship was observed for the short and long phantom doses listed in Table 3 and Fig. 8; therefore, such correction factors can be potentially used in the $\mathrm{f}$ (0) ${ }^{\mathrm{CB}}{ }_{\mathrm{w}}$ method.

In this study, CBCT applications for dental and some TrueBeam scanning modes (Half-Fan mode and a full scan $\left(360^{\circ}\right)$ ) [5] were not performed to determine the devised $\mathrm{f}(0)^{\mathrm{CB}}{ }_{\mathrm{w}}$ method. The $\mathrm{f}(0){ }^{\mathrm{CB}}{ }_{\mathrm{w}}$ method is limited and was devised under the following conditions: (1) partial angle irradiations, (2) rotating center corresponding to phantom center, and (3) beam width of lateral direction in symmetry. Additional studies are needed to verify whether the devised method is congruous for other conditions.

The two methods (kerma-area product (KAP) and dose to the field of view ( $\left.\mathrm{D}_{\mathrm{FOV}}\right)$ ) were proposed for CBCT radiation output index by the European Federation of Organisations for Medical Physics (EFOMP), European Society for Radiotherapy and Oncology (ESTRO), and International Atomic Energy Agency (IAEA) [22]. As described above, the $\mathrm{CTDI}_{\mathrm{IEC}}$ and point dose approaches are also CBCT dose metrics. The idea of using a specific dose index for CBCT dosimetry is controversial, and to date there is no dose index agreed on globally for CBCT. We propose the devised $\mathrm{f}(0)^{\mathrm{CB}}{ }_{\mathrm{w}}$ method as one way to evaluate CBCT dose accurately, and the results of this study would be useful for the future of CBCT dosimetry.

\section{Conclusions}

In this work, a new dose metric $\left(\mathrm{f}(0)^{\mathrm{CB}}{ }_{\mathrm{w}}\right)$ was devised for CBCT dosimetry to provide a more accurate average dose by performing Monte Carlo simulations and using the linear least-squares technique. The $f$ $(0)^{\mathrm{CB}}$ w value was calculated by averaging four "point doses" at $90^{\circ}$ intervals and the optimal radial positions of the PMMA cylindrical phantom. The developed $\mathrm{f}(0)^{\mathrm{CB}}{ }_{\mathrm{w}}$ method can estimate the average dose with high accuracy corresponding to a $4.1 \%$ error over a wide range of phantom diameters. In addition, this technique is very robust for CBCT dosimetry because the optimal radial positions are independent of the 


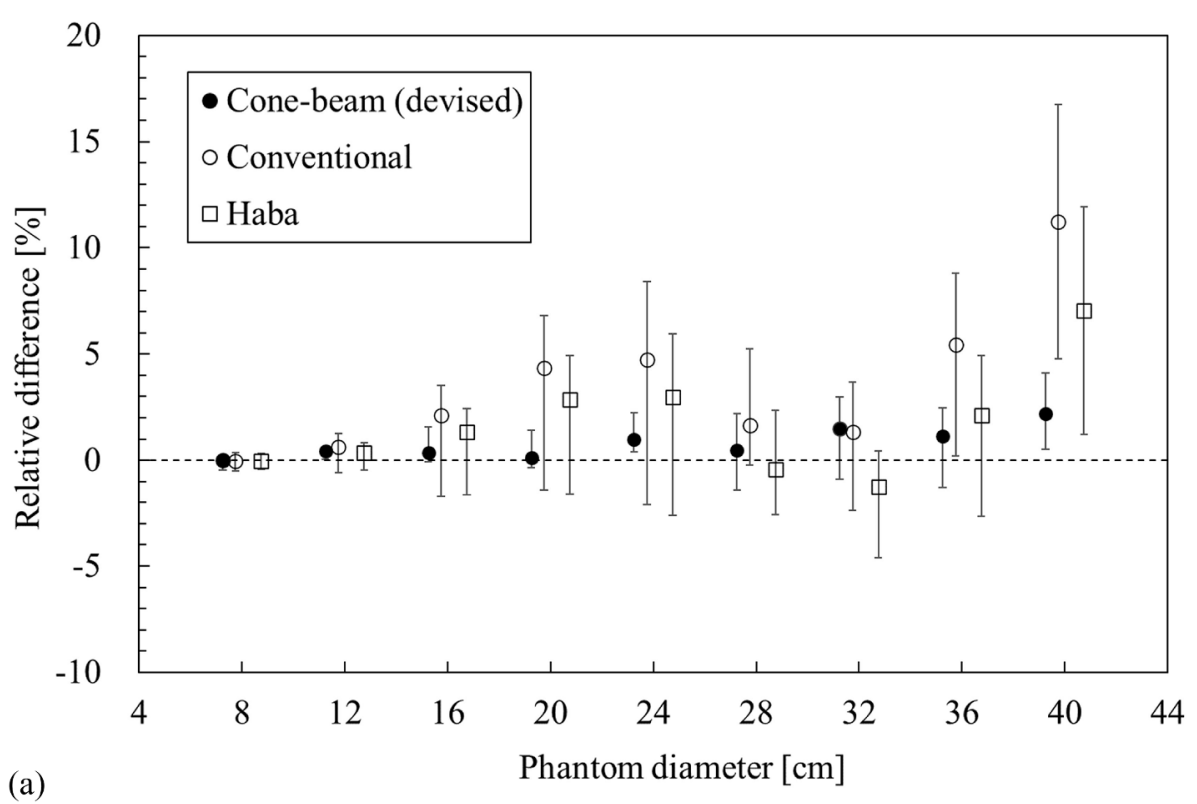

(a)

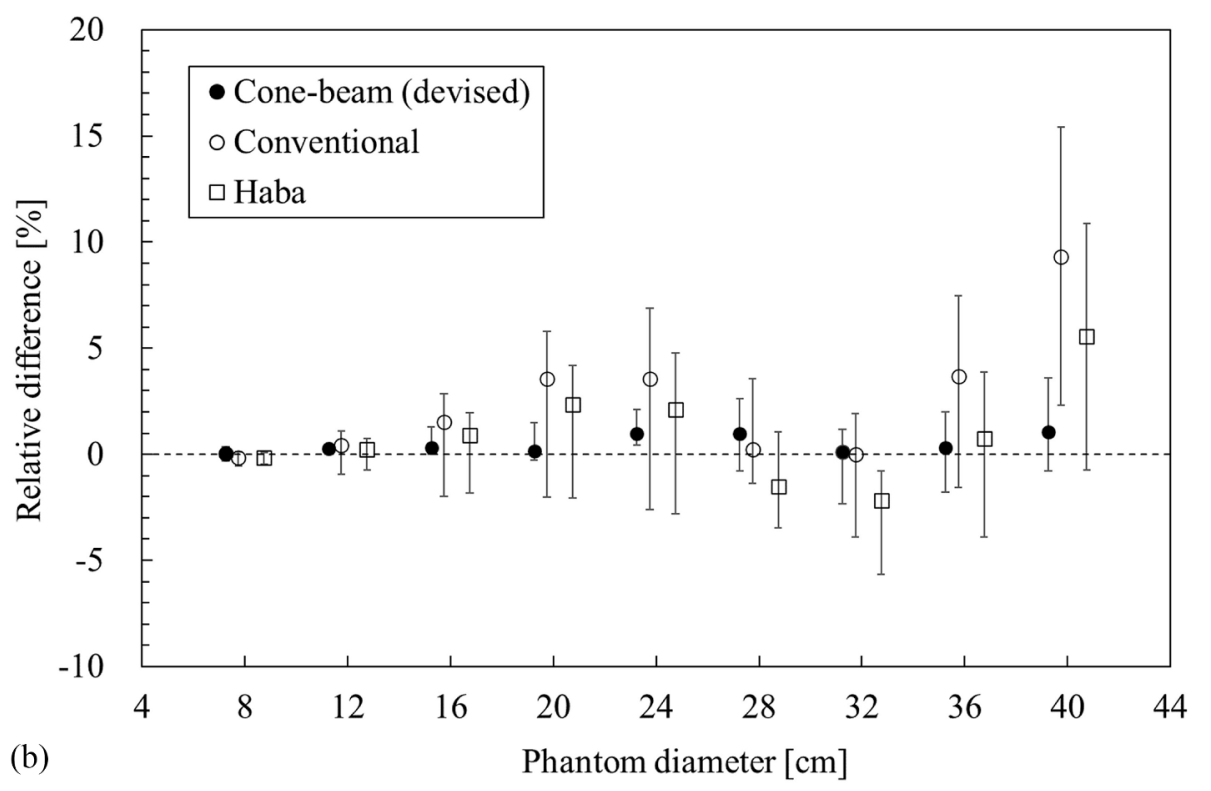

Fig. 8. Relative differences between the $\bar{D}_{\text {cs }}$ and each dose index value obtained at phantom lengths of (a) 15 and (b) $45 \mathrm{~cm}$. In the legend, "Cone-beam (devised)," "Conventional," and "Haba" indicate the $\mathrm{f}$ (0) ${ }^{\mathrm{CB}}{ }_{\mathrm{w}}, \mathrm{CTDI}^{\mathrm{C}}{ }_{\mathrm{w}}$, and $\mathrm{CTDI}^{\mathrm{H}}{ }_{\mathrm{w}}$ methods, respectively. Each symbol denotes the relative difference averaged for all CBCT scan protocols listed in Table 1, and each error bar indicates its maximum and minimum magnitudes. The horizontal broken line corresponds to the relative difference of $0 \%$, at which each dose index value equals the average phantom dose.
CBCT scan protocol (scanner type, rotation angle, and tube voltage) and phantom length $(L=15$ and $45 \mathrm{~cm})$. Hence, the devised $\mathrm{f}(0){ }^{\mathrm{CB}}{ }_{\mathrm{w}}$ method is more suitable for CBCT dosimetry than the previously developed $\mathrm{CTDI}_{\mathrm{w}}\left(\mathrm{CTDI}^{\mathrm{C}}{ }_{\mathrm{w}}\right.$ and $\left.\mathrm{CTDI}^{\mathrm{H}}{ }_{\mathrm{w}}\right)$ metrics.

\section{Declaration of Competing Interest}

The authors declare that they have no known competing financial interests or personal relationships that could have appeared to influence the work reported in this paper.

\section{Acknowledgment}

This work was supported by JSPS KAKENHI (grant number: JP19K17282).

\section{References}

[1] AAPM Task Group 23. The measurement, reporting, and management of radiation dose in CT. AAPM Rep No 962008.
[2] Fahrig R, Dixon R, Payne T, Morin RL, Ganguly A, Strobel N. Dose and image quality for a cone-beam C-arm CT system: Cone-beam C-arm CT system. Med. Phys. 2006;33(12):4541-50. https://doi.org/10.1118/1.2370508.

[3] IEC 60601-2-44. Medical electrical equipment-Part 2-44: Particular requirements for the basic safety and essential performance of X-ray equipment for computed tomography. IEC 2009.

[4] AAPM Task Group 111. Comprehensive methodology for the evaluation of radiation dose in X-ray computed tomography. AAPM Rep No 1112010.

[5] Abuhaimed A, J Martin C, Sankaralingam M, J Gentle D, McJury M. An assessment of the efficiency of methods for measurement of the computed tomography dose index (CTDI) for cone beam (CBCT) dosimetry by Monte Carlo simulation. Phys Med Biol 2014;59(21):6307-26. https://doi.org/10.1088/0031-9155/59/21/ 6307.

[6] Abuhaimed A, Martin CJ, Sankaralingam M, Gentle DJ. A Monte Carlo investigation of cumulative dose measurements for cone beam computed tomography (CBCT) dosimetry. Phys Med Biol 2015;60(4):1519-42. https://doi org/10.1088/0031-9155/60/4/1519.

[7] Dixon RL. A new look at CT dose measurement: Beyond CTDI. Med Phys 2003;30 (6):1272-80. https://doi.org/10.1118/1.1576952.

[8] Li X, Zhang Da, Yang J, Liu B. A study of the short- to long-phantom dose ratios for CT scanning without table translation: Short- to long-phantom CT dose ratios for stationary-table modes. Med Phys 2014;41(9):091912. https://doi.org/10.1118/ 1.4893753 . 
[9] Kim S, Alaei P. Implementation of full/half bowtie filter models in a commercial treatment planning system for kilovoltage cone-beam CT dose estimations. J App Clin Med Phys 2016;17(2):153-64. https://doi.org/10.1120/jacmp.v17i2.5988.

[10] Leitz W, Axelsson B, Szendrö G. Computed tomography dose assessment - a practical approach. Radiat Prot Dosimetry 1995;57:377-80. https://doi.org/ 10.1093/rpd/57.1-4.377.

[11] Bakalyar DM. A critical look at the numerical coefficients in CTDIvol. Med Phys 2006;33:2003.

[12] Haba T, Koyama S, Kinomura Y, Ida Y, Kobayashi M. New weighting factor of weighted CTDI equation for PMMA phantom diameter from 8 to $40 \mathrm{~cm}$ : A Monte Carlo study. Med Phys 2017;44(12):6603-9. https://doi.org/10.1002/mp.12601.

[13] AAPM Task Group 204. Size-specific dose estimates (SSDE) in pediatric and adult body CT examinations. AAPM Rep No 2042011.

[14] McMillan K, McNitt-Gray M, Ruan D. Development and validation of a measurement-based source model for kilovoltage cone-beam CT Monte Carlo dosimetry simulations: Development and validation of a measurement-based CBCT source model. Med Phys 2013;40(11):111907. https://doi.org/10.1118/ 1.4823795 .

[15] Tucker DM, Barnes GT, Chakraborty DP. Semiempirical model for generating tungsten target x-ray spectra: Model for tungsten target x-ray spectra. Med Phys 1991;18(2):211-8. https://doi.org/10.1118/1.596709.
[16] Turner AC, Zhang Di, Kim HJ, DeMarco JJ, Cagnon CH, Angel E, et al. A method to generate equivalent energy spectra and filtration models based on measurement for multidetector CT Monte Carlo dosimetry simulations: Generation of source models for MDCT Monte Carlo. Med Phys 2009;36(6Part1):2154-64. https://doi.org/ 10.1118/1.3117683.

[17] Bakalyar D, Feng W, McKenney S. SU-G-206-06: analytic dose function for CT scans in infinite cylinders as a function of scan length and cylinder radius. Med Phys 2016;43:3640. https://doi.org/10.1118/1.4956947.

[18] Boone JM. Method for evaluating bow tie filter angle-dependent attenuation in CT: Theory and simulation results: Bow tie filter characterization in CT. Med Phys 2010;37(1):40-8. https://doi.org/10.1118/1.3264616.

[19] AAPM Task Group 200. The design and use of the ICRU/AAPM CT radiation dosimetry phantom : An implementation of AAPM report 111. AAPM Rep No 200 2020.

[20] AAPM Task Group 220. Use of Water Equivalent Diameter for Calculating Patient Size and Size-Specific Dose Estimates (SSDE) in CT. AAPM Rep No 2202014.

[21] IEC. 62985. Methods for calculating size specific dose estimates (SSDE) for computed tomography. IEC 2019.

[22] de las Heras Gala Hugo, Torresin Alberto, Dasu Alexandru, Rampado Osvaldo, Delis Harry, Giron Irene Hernandez, et al. Quality control in cone-beam computed tomography (CBCT) EFOMP-ESTRO-IAEA protocol (summary report). Phys Med 2017;39:67-72. https://doi.org/10.1016/j.ejmp.2017.05.069. 